\title{
Diffraction from Polygonal-Conical Screens, An Operator Approach ${ }^{\dagger}$
}

\author{
Luís P. Castro, Roland Duduchava and Frank-Olme Speck
}

To Professor António Ferreira dos Santos with gratitude

\begin{abstract}
The aim of this work is to construct explicitly resolvent operators for a class of boundary value problems in diffraction theory. These are formulated as boundary value problems for the three-dimensional Helmholtz equation with Dirichlet or Neumann conditions on a plane screen of polynomial-conical form (including unbounded and multiplyconnected screens), in weak formulation. The method is based upon operator theoretical techniques in Hilbert spaces, such as the construction of matrical coupling relations and certain orthogonal projections, which represent new techniques in this area of applications. Various cross connections are exposed, particularly considering classical WienerHopf operators in Sobolev spaces as general Wiener-Hopf operators in Hilbert spaces and studying relations between the crucial operators in game. Former results are extended, particularly to multiply-connected screens.
\end{abstract}

Mathematics Subject Classification (2010). Primary 78A45; Secondary 47G30.

Keywords. Diffraction, plane screen, polygonal domain, conical domain, Dirichlet problem, Neumann problem, explicit solution, Wiener-Hopf operator, Sobolev space, matrical coupling, orthogonal projector.

\footnotetext{
This work was started during the Workshop on Operator Theory and Operator Algebras in September 2012 at Lisbon.

$\dagger$ Accepted author's manuscript (AAM) published in [Operator Theory, Operator Algebras and Applications, Operator Theory: Advances and Applications 242 (2014), 113137] [DOI: 10.1007/978-3-0348-0816-3_6] The final publication is available at Springer via http://link.springer.com/chapter/10.1007/978-3-0348-0816-3_6
} 


\section{Formulation of problems and main results}

Given a proper open subset $\Sigma \subset \mathbb{R}^{2}$, we consider the domain $\Omega$ defined by

$$
\begin{aligned}
& \Omega=\mathbb{R}^{3} \backslash \Gamma \\
& \Gamma=\bar{\Sigma} \times 0=\left\{x=\left(x_{1}, x_{2}, 0\right) \in \mathbb{R}^{3}: x^{\prime}=\left(x_{1}, x_{2}\right) \in \bar{\Sigma}\right\} .
\end{aligned}
$$

For convenience the boundary manifold $\Gamma$ is closed (in $\mathbb{R}^{3}$ ) and the screen $\Sigma$ is open (a domain in $\mathbb{R}^{2}$ ). However, the two sets will be identified in some sense (provided int $\operatorname{clos} \Sigma=\Sigma$ ). As a rule we assume $\Sigma \neq \emptyset, \Sigma \neq \mathbb{R}^{2}$, if nothing else is said.

Problems of diffraction from a plane screen $\Gamma$ are often formulated in terms of (or reduced to) the solution of the three-dimensional Helmholtz equation (HE) in $\Omega$ with Dirichlet or Neumann conditions on $\Gamma$, briefly written as

$$
\begin{array}{ll}
\left(\Delta+k^{2}\right) u & =0 \quad \text { in } \Omega \\
B u & =g \text { on } \Gamma=\partial \Omega .
\end{array}
$$

Herein $k$ is the wave number and we assume that $\Im m k>0$ throughout this paper (some parts are restricted to $\Re e k=0$ ). $B$ stands for the boundary operator, taking the trace or normal derivative of $u$ on $\Gamma$. We think of the weak formulation looking for $u \in L^{2}(\Omega)$ with restrictions $u^{ \pm}=\left.u\right|_{\Omega^{ \pm}}$to the upper and lower half-space $\Omega^{ \pm}=\left\{x \in \mathbb{R}^{3}: \pm x_{3}>0\right\}$ that satisfy $u^{ \pm} \in H^{1}\left(\Omega^{ \pm}\right)$ and the common transmission conditions across the complement of $\bar{\Sigma}$ :

$$
\Sigma^{\prime}=\mathbb{R}^{2} \backslash \bar{\Sigma},
$$

namely

$$
\begin{array}{ll}
u_{0}^{+}-u_{0}^{-}=\left.\left[u^{+}-u^{-}\right]\right|_{x_{3}=0} & =0 \\
u_{1}^{+}-u_{1}^{-}=\left.\left[\partial u^{+} / \partial x_{3}-\partial u^{-} / \partial x_{3}\right]\right|_{x_{3}=0}=0
\end{array}
$$

according to the trace theorem and by help of representation formulas, see [20] and Section 2 for details. In a sense, this is equivalent to state that the HE holds across the complementary screen $\Sigma^{\prime}[26]$. The boundary data $g$ are arbitrarily given in the corresponding data space $H^{1 / 2}(\Sigma)$ or $H^{-1 / 2}(\Sigma)$, respectively (values of $g$ in the boundary of $\Sigma$ do not matter in this space setting).

For convenience we study the (homogeneous) HE, since boundary value problems for the inhomogeneous HE $A u=\left(\Delta+k^{2}\right) u=f$ can be "equivalently reduced" under the present assumptions, see [40]. Hence the operator associated with the boundary value problem (BVP) can be written as

$$
B_{0}=\left.B\right|_{\text {ker } A}: \mathcal{H}^{1}(\Omega) \rightarrow H^{ \pm 1 / 2}(\Sigma)
$$

where $\mathcal{H}^{1}(\Omega)$ denotes the space of weak solutions of the HE in $\Omega$ and $B_{0}$ denotes the restriction of $B$ to this space. We are looking for the inverse $B_{0}^{-1}$, the so-called resolvent operator.

Sometimes different data $g^{ \pm}$are prescribed on the two banks $\Sigma^{ \pm}$of the screen corresponding to $x_{3}= \pm 0$. This generalization is not very important 
from the physical point of view (where $g$ denotes the trace of the "incoming field", e.g.), but useful for understanding the structure of the problems. In this case the BVPs can be briefly written in the form

$$
\begin{aligned}
& u \in \mathcal{H}^{1}(\Omega) \\
& B_{0} u=\left(\begin{array}{c}
B^{+} \\
B^{-}
\end{array}\right) u=g=\left(\begin{array}{l}
g^{+} \\
g^{-}
\end{array}\right) \text {on } \Gamma=\partial \Omega
\end{aligned}
$$

where now

- in case of the Dirichlet problem: $g$ is given in the space $H^{1 / 2}(\Sigma)^{2}=$ $H^{1 / 2}(\Sigma) \times H^{1 / 2}(\Sigma)$ with the compatibility condition that $g^{+}-g^{-}$is extensible by zero from $\Sigma$ to the full plane (corresponding to $x_{3}=0$ ) within $H^{1 / 2}\left(\mathbb{R}^{2}\right)$,

- in case of the Neumann problem: $g$ is given in the space $H^{-1 / 2}(\Sigma)^{2}=$ $H^{-1 / 2}(\Sigma) \times H^{-1 / 2}(\Sigma)$ with the compatibility condition that $g^{+}-g^{-}$be extensible by zero from $\Sigma$ to the full plane (corresponding to $x_{3}=0$ ) within $H^{-1 / 2}\left(\mathbb{R}^{2}\right)$, see [20] for details.

We express these compatibility conditions briefly by writing

$$
g \in H^{1 / 2}(\Sigma)_{\sim}^{2} \text { respectively } g \in H^{-1 / 2}(\Sigma)_{\sim}^{2} .
$$

In several publications the second compatibility condition is written in the form $g^{+}+g^{-} \in \widetilde{H}^{-1 / 2}(\Sigma)$ according to the convention that the normal derivative is always taken with respect to the outer (or inner) normal, i.e., $g^{-}=-\partial / \partial x_{3} u$ on $\Sigma^{-}$, in contrast to the present situation.

The question of "low regularity", i.e., $\left.u \in H^{1+\varepsilon}, \varepsilon \in\right] 0,1 / 2[$, could be included from the beginning, but will be answered only at the end of Section 5 , to keep the notation short.

It is well known that all the above-mentioned BVPs are correctly posed provided $\Sigma$ is a strong Lipschitz domain (bounded) or special Lipschitz domain (unbounded) [20, 42]. This results from the use of Green's formula (for uniqueness), reduction to boundary integral or pseudo-differential equations, their Fredholm property, an index formula and strong ellipticity (for existence). The fact that the associated operator $B_{0}: \mathcal{H}^{1}(\Omega) \rightarrow H^{ \pm 1 / 2}(\Sigma)$ is a bounded, linear and bijective operator acting in Hilbert spaces, implies (by the inverse mapping theorem) that $B_{0}^{-1}$ is continuous.

The question is: Can we obtain an explicit formula of $B_{0}^{-1}$, not only for very special screens like half-planes and certain cones [25]? In the present article we shall admit solutions in closed analytical form or series expansion, as well as infinite operator products which are strongly convergent. In this sense, the answer will be positive for a surprisingly large class of problems and possibly not useless in view of the capacity of modern computers.

Let us briefly look at the classes of domains $\Sigma \subset \mathbb{R}^{2}$ under consideration. The following domain properties are crucial in what follows.

- First we shall assume the strong extension property [20, 23], i.e., for any $s \in \mathbb{R}$, there exists a continuous extension operator which is left 
invertible by restriction:

$$
\begin{aligned}
\ell_{\Sigma}^{s} & : H^{s}(\Sigma) \rightarrow H^{s}\left(\mathbb{R}^{2}\right) \\
r_{\Sigma} \ell_{\Sigma}^{s} & =I_{H^{s}(\Sigma)} .
\end{aligned}
$$

Lipschitz domains (that are bounded and characterized by fulfilling the uniform cone property $[18,20])$ and (unbounded) special Lipschitz domains in the sense of [42] (of the form $\Sigma=\left\{\left(x_{1}, x_{2}\right) \in \mathbb{R}^{2}: x_{2}>\varphi\left(x_{1}\right)\right\}$ where $\varphi$ is uniformly Lipschitz continuous and rotations of this kind of domain) fulfil the strong extension property. The existence of continuous extension operators (1.7) guarantees the equivalence of $B_{0}$ to operators which have the form of a general Wiener-Hopf operator $W$ (see Section 2), i.e., $B_{0}=E W F$ where $E$ and $F$ are linear homeomorphisms.

- Second we shall confine our considerations to domains with the property int $\operatorname{clos} \Sigma=\Sigma$, which is needed for a relaxed use of Sobolev spaces. Note that this excludes "cracks" in the screen (also called "slit domains") with discontinuities across the cracks, which could be considered using more complicated notation than $H^{s}(\Sigma)$ (in general, the notion of $H^{s}(\Sigma)$ with Lipschitz domains $\Sigma$ is not suitable for that case, see [20], p. 110, and the introduction of [15], for instance).

A domain $\Sigma$ with these two properties is said to be an E-domain. The properties are actually needed only for $s= \pm 1 / 2$ in the basic results.

Further we shall work with an algebra $\mathcal{A}_{2}$ of open subsets $\Sigma \subset \mathbb{R}^{2}$ which contains open half-planes, finite intersections and the interior of complements of elements of $\mathcal{A}_{2}$.

Therefore we introduce the following.

Definition 1.1. A convex polygonal-conical domain (convex PCD) in $\mathbb{R}^{2}$ is given by

$$
\Sigma=\bigcap_{j=1, \ldots, m} \Sigma_{j} \quad \text { where } \Sigma_{j} \quad \text { are open half-planes. }
$$

A polygonal-conical domain $(\mathrm{PCD})$ in $\mathbb{R}^{2}$ is given by

$$
\Sigma=\operatorname{int} \bigcup_{j=1, \ldots, m} \operatorname{clos} \Sigma_{j}
$$

where $\Sigma_{j}$ are convex PCDs which do not meet in a corner.

Remark 1.2. The following observations are obvious:

1. Convex PCDs are simply connected, PCDs may be multiply connected, both possibly unbounded (cones are included).

2. PCDs are E-domains.

3. The set of PCDs (including $\Sigma=\emptyset$ and $\Sigma=\mathbb{R}^{2}$ ) coincides with the minimal set algebra $\mathcal{A}_{2}$ described above, since they allow a representation

$$
\Sigma=\mathbb{R}^{2} \backslash\left(\bigcap_{j=1, \ldots, m}\left(\mathbb{R}^{2} \backslash \Sigma_{j}\right)\right)
$$


where $\Sigma_{j}$ are convex PCDs. This results from the De Morgan formulas and some elementary topological consideration.

4. The set of Lipschitz domains does not form a set algebra, because the intersection of two Lipschitz domains does not necessarily have the strong extension property. Also special Lipschitz domains do not generate an algebra of sets which have the strong extension property, for the same reason.

In order to describe the spaces for the boundary data in more detail, we recall the definition of the usual Sobolev spaces $H^{s}=H^{s}\left(\mathbb{R}^{n}\right)$ (sometimes named Bessel potential or fractional Sobolev spaces) and of the Sobolev spaces $H^{s}(\Sigma), H_{\Sigma}^{s}, \widetilde{H}^{s}(\Sigma)$, as well (see, e.g., [16, 20]). Thus let

$$
H^{s}=H^{s}\left(\mathbb{R}^{n}\right)=\left\{f \in \mathcal{S}^{\prime}:\|f\|=\left(\int_{\mathbb{R}^{n}}|\hat{f}(\xi)|^{2}\left(\xi^{2}+1\right)^{s} d \xi\right)^{1 / 2}<\infty\right\}
$$

where $\xi^{2}$ stands for $|\xi|^{2}$ and $\mathcal{S}^{\prime}=\mathcal{S}^{\prime}\left(\mathbb{R}^{n}\right)$ denotes the Schwartz distribution space and $\hat{f}(\xi)=\mathcal{F}_{x \mapsto \xi} f(x)=\int_{\mathbb{R}^{n}} e^{i x \xi} f(x) d x$ the (n-dimensional) Fourier transform of $f \in \mathcal{S}$ extended to distributions $f \in \mathcal{S}^{\prime} . H^{s}$ is a Hilbert space with inner product

$$
\langle\varphi, \psi\rangle_{s}=\int_{\mathbb{R}^{n}} \hat{\varphi}(\xi) \overline{\hat{\psi}(\xi)}\left(\xi^{2}+1\right)^{s} d \xi \quad, \quad \varphi, \psi \in H^{s}\left(\mathbb{R}^{n}\right) .
$$

The function $\lambda(\xi)=\left(\xi^{2}+1\right)^{1 / 2}, \xi \in \mathbb{R}^{n}$, will play a special role in what follows, since it can be considered as a particular case of the square root of the "Helmholtz symbol" $t(\xi)=\lambda_{k}(\xi)=\left(\xi^{2}-k^{2}\right)^{1 / 2}$ for $k=i$ (the double notation has historical reasons). We shall always choose branches, continuous in $\mathbb{R}^{n}$, such that $\lambda_{k}(\xi) \rightarrow+\infty$ as $|\xi| \rightarrow+\infty$. It may be useful to consider the spaces $H^{s}$ as the isometric images of the Bessel potential operators

$$
\Lambda^{-s}=\mathcal{F}^{-1} \lambda^{-s} \cdot \mathcal{F}: L^{2} \rightarrow H^{s} \quad, \quad s \in \mathbb{R} .
$$

The restriction operator which restricts a function or distribution on $\mathbb{R}^{n}$ to an open subset $\Sigma$ will be denoted by $r_{\Sigma}$. Thus $H^{s}(\Sigma)=r_{\Sigma}\left(H^{s}\right)$, and the norm in $H^{s}(\Sigma)$ is defined by

$$
\|f\|_{H^{s}(\Sigma)}=\inf _{\ell}\|\ell f\|_{H^{s}}
$$

where $\ell f$ stands for any extension of $f$ to a distribution in $H^{s}$. An equivalent norm can be defined via the Sobolev-Slobodetski norm for $s>0$ and via a duality for $s<0$. Furthermore, we denote by $H_{\Sigma}^{s}$ the (closed) subspace of $H^{s}$ which consists of all distributions with support in the closure of $\Sigma$. By $\widetilde{H}^{s}(\Sigma)$ we denote the space of all distributions which are the restrictions of distributions in $H_{\Sigma}^{s}$, i.e., $\widetilde{H}^{s}(\Sigma)=r_{\Sigma}\left(H_{\Sigma}^{s}\right)$. A norm is defined by

$$
\|f\|_{\widetilde{H}^{s}(\Sigma)}=\inf _{\ell_{0}}\left\|\ell_{0} f\right\|_{H^{s}}
$$

where $\ell_{0} f$ stands for any extension of $f$ to a distribution in $H_{\Sigma}^{s}$ (which is unique only for $s \geq-1 / 2$, see [14], pages $4-5$, in which case the last infimum 
is redundant). Notice that while $\widetilde{H}^{s}(\Sigma)$ is always continuously embedded in $H^{s}(\Sigma)$, these two spaces coincide for $\left.s \in\right]-1 / 2,1 / 2[$. In various publications $\widetilde{H}^{s}(\Sigma)$ is defined as the set of $H^{s}(\Sigma)$ function(al)s that are extendible by zero to an element of $H^{s}\left(\mathbb{R}^{n}\right)[18,20]$. For E-domains this definition is equivalent to the present one.

Now we are in a position to summarize the main result.

Theorem 1.3 (Main Theorem). Let $\Sigma$ be a PCD. Then the resolvent operator $B_{0}^{-1}$ (see (1.4), (1.5)) for the Dirichlet or Neumann problem is explicitly given in terms of infinite operator products (presented in Sections 4 and 5) which strongly converge in the common (Bessel potential) norm of $H^{ \pm 1 / 2}\left(\mathbb{R}^{2}\right)$ for $k=i$ and in a modified equivalent norm for $k \in i \mathbb{R}_{+}$, respectively. In the remaining cases of $k \in \mathbb{C}, \Im m k>0$ the resolvent operator can be explicitly represented by (additional) use of Neumann series.

The principle steps are: (1) to show operator equivalence of $B_{0}$ with a boundary pseudo-differential operator that has the form of a general WienerHopf operator (WHO), (2) to represent $B_{0}^{-1}$ in terms of a certain projector acting in $H^{ \pm 1 / 2}\left(\mathbb{R}^{2}\right)$, which depends heavily on the form of $\Sigma$, (3) for screens which are convex PCDs, to give an explicit formula for these kind of projectors in case of $k \in i \mathbb{R}_{+}$, choosing a topology where they are orthogonal and using a result of Halmos [19] for the representation of the orthogonal projector onto the intersection of closed Hilbert subspaces, (4) to reduce the case of arbitrary $k$ with $\Im m k>0$ to the previous by approximation, and finally (5) to reduce the case of non-convex screens to the case of convex screens by matrical coupling of associated WHOs and the so-called geometric perspective of Ferreira dos Santos [30, 31] for general WHOs, noting that not only complements of convex screens are admitted, but arbitrary PCDs.

Some similar ideas appeared already in special situations or different settings, see [15, 24, 25, 37, 39] and will be pointed out in the corresponding context. However, some of the cited results are presented here with a new, more compact proof, e.g., Theorem 3.5 and parts of Theorem 3.8 and of Theorem 3.10.

From the historical point of view, one can say, that the story started with the solution of Sommerfeld's half-plane problem [35] by modern WienerHopf methods [26], contributions to the diffraction by a quarter-plane [11, $12,25,44]$ and the discovery of relations with general WHOs [31, 37]. The present paper could be regarded as an extension of [24] to non-convex, general polynomial-conical screens, however with several new techniques that provide a deeper insight into the structure of this kind of BVPs. Finally it should be noticed that the present screen problems are quite different from wedge diffraction problems in formulation and structure. 


\section{Reduction to boundary pseudo-differential equations and form of resolvent operators}

For this step we need a precise notation of Wiener-Hopf operators in Sobolev spaces. We shall use only a scalar version (the matrix analogue is evident).

Definition 2.1. A Wiener-Hopf operator in Sobolev spaces (briefly referred to as classical $\mathrm{WHO}$ ) is given by

$$
W_{\phi, \Sigma}=r_{\Sigma} A_{\phi} \quad: \quad H_{\Sigma}^{r} \rightarrow H^{s}(\Sigma)
$$

where $\Sigma \subset \mathbb{R}^{n}$ is a domain, $r, s \in \mathbb{R}, A_{\phi}=\mathcal{F}^{-1} \phi \cdot \mathcal{F}$ is a convolution (translation invariant) operator in $\mathbb{R}^{n}$ of order $r-s$, i.e., $\phi_{0}=\phi \lambda^{r-s} \in$ $L^{\infty}\left(\mathbb{R}^{n}\right)$.

Remark 2.2. Other popular notations of WHOs are the following. The "classical WHO" acting on $L^{2}\left(\mathbb{R}_{+}\right)$(or on $L^{p}\left(\mathbb{R}_{+}\right)$etc. [22]) is given by

$$
W f(x)=a f(x)+\int_{0}^{\infty} k(x-y) f(y) d y \quad, \quad x>0
$$

with $a \in \mathbb{C}, k \in L^{1}(\mathbb{R})$. It can be briefly written as

$$
W \quad=\quad r_{+} A_{\phi} \ell_{0} \quad: \quad L^{2}\left(\mathbb{R}_{+}\right) \rightarrow L^{2}\left(\mathbb{R}_{+}\right)
$$

where $\phi=a+\mathcal{F} k$ is the Fourier symbol of $W[17,29]$ and $\ell_{0}$ the zero extension from $L^{2}\left(\mathbb{R}_{+}\right)$to $L^{2}(\mathbb{R})$. This is easily generalized to an operator acting on $L^{2}(\Sigma), \Sigma \subset \mathbb{R}^{n}$ by writing

$$
W=r_{\Sigma} A_{\phi} \ell_{0} \quad: \quad L^{2}(\Sigma) \rightarrow L^{2}(\Sigma)
$$

and makes sense already, if $\Sigma$ is measurable. The direct generalization to Sobolev spaces makes sense if the extension $\ell_{0}: H^{s}(\Sigma) \rightarrow H^{s}\left(\mathbb{R}^{n}\right)$ is well defined, e.g., for Lipschitz or special Lipschitz domains $\Sigma$. It can be written as

$$
W=r_{\Sigma} A_{\phi} \ell_{0} \quad: \quad H^{s}(\Sigma) \rightarrow H^{s}(\Sigma)
$$

defined by restriction $(s>0)$ or by continuous extension $(s<0)$, if $s \in$ ] $-1 / 2,1 / 2$ [. In contrast, the "Eskin like notation" (2.1) makes sense for all $s \in \mathbb{R}$ and arbitrary domains $\Sigma[16,28]$.

Another generalization will be important for our purposes, the notion of "general WHOs". That will be discussed in Section 3.

Now we come to the point where these operators appear in reality.

Theorem 2.3 (Representation Theorem for the Dirichlet problem). Assume that $\Sigma \subset \mathbb{R}^{2}$ be any (proper) open subset of $\mathbb{R}^{2}, \Omega$ be given by (1.1) and $\Omega^{ \pm}=\left\{x \in \mathbb{R}^{3}: \pm x_{3}>0\right\}$. Then the Dirichlet problem in $\Omega$ (see (1.5)) is well-posed if and only if the following WHO is invertible:

$$
W_{t^{-1}, \Sigma}=r_{\Sigma} A_{t^{-1}} \quad: \quad H_{\Sigma}^{-1 / 2} \rightarrow H^{1 / 2}(\Sigma) .
$$

In this case, the solution of the Dirichlet problem is given by the formulas

$$
u=\mathcal{K}_{D, \Omega}\left(g_{1}, g_{2}\right)=\left\{\begin{array}{lll}
\mathcal{K}_{D, \Omega^{+}} u_{0}^{+} & \text {in } & \Omega^{+} \\
\mathcal{K}_{D, \Omega^{-}} u_{0}^{-} & \text {in } & \Omega^{-}
\end{array}\right.
$$




$$
\begin{aligned}
& \mathcal{K}_{D, \Omega^{+}} u_{0}^{+}(x)=\mathcal{F}_{\xi^{\prime} \mapsto x^{\prime}}^{-1} e^{-t\left(\xi^{\prime}\right) x_{3}} \widehat{u_{0}^{+}}\left(\xi^{\prime}\right) \\
&=\frac{1}{(2 \pi)^{2}} \int_{\mathbb{R}^{2}} e^{-i \xi^{\prime} x^{\prime}-t\left(\xi_{1}, \xi_{2}\right) x_{3}} \widehat{u_{0}^{+}}\left(\xi_{1}, \xi_{2}\right) d \xi^{\prime} \\
&=\frac{1}{(2 \pi)^{2}} \int_{\mathbb{R}^{2}} e^{-i \xi^{\prime} x^{\prime}+t\left(\xi_{1}, \xi_{2}\right) x_{3}} \widehat{u_{0}^{-}}\left(\xi_{1}, \xi_{2}\right) d \xi^{\prime} \\
&\left(\begin{array}{l}
u_{0}^{+} \\
u_{0}^{-}
\end{array}\right)=\Upsilon_{D}^{-1}\left(\begin{array}{cc}
\ell_{0} & \mathcal{F}_{\xi^{\prime} \mapsto x^{\prime}}^{-1} e^{t\left(\xi^{\prime}\right) x_{3}} \widehat{u_{0}^{-}}\left(\xi^{\prime}\right) \\
0 & A_{t^{-1}} W_{t^{-1}, \Sigma}^{-1}
\end{array}\right) \Upsilon_{D}\left(\begin{array}{l}
g^{+} \\
g^{-}
\end{array}\right), \quad \Upsilon_{D}=\left(\begin{array}{cc}
I & -I \\
I & I
\end{array}\right)
\end{aligned}
$$

abbreviating $\xi^{\prime}=\left(\xi_{1}, \xi_{2}\right) \in \mathbb{R}^{2}, d \xi^{\prime}=d \xi_{1} d \xi_{2}, t\left(\xi^{\prime}\right)=\left(\xi_{1}^{2}+\xi_{2}^{2}-k^{2}\right)^{1 / 2}$, $x^{\prime}=\left(x_{1}, x_{2}\right) \in \mathbb{R}^{2}$ and $\xi^{\prime} x^{\prime}=\xi_{1} x_{1}+\xi_{2} x_{2}$.

Proof. (Sketch) Based on ideas from [24] and [15] we see that a possible solution is represented by the trace data $u_{0}^{ \pm}$(in the entire plane $x_{3}=0$ ), where $u_{0}^{+}-u_{0}^{-}$is directly given and that $u_{0}^{+}+u_{0}^{-}=A_{t}^{-1}\left(u_{1}^{+}-u_{1}^{-}\right)$where $u_{1}^{+}-u_{1}^{-}$satisfies a boundary pseudo-differential equation which has the form of a (generalized) Wiener-Hopf equation on $\Sigma$. If the WHO is invertible, the BVP is well-posed, since one can verify that the formulas represent linear homeomorphisms between the data and the solution spaces. Conversely, if the BVP is well-posed, the WHO must be bijective. As a bounded linear operator, it is necessarily a homeomorphism according to the inverse mapping theorem.

More details can be found in the context of Sommerfeld potentials (where $\Sigma$ is a half-plane) [14, 15]. Also BVPs for the Lamé equation have been solved in a similar way already in [13].

Theorem 2.4 (Representation Theorem for the Neumann problem). Assume that $\Sigma \subset \mathbb{R}^{2}$ be any (proper) open subset of $\mathbb{R}^{2}, \Omega$ be given by (1.1) and $\Omega^{ \pm}=\left\{x \in \mathbb{R}^{3}: \pm x_{3}>0\right\}$. Then the Neumann problem in $\Omega$ (see (1.5)) is well-posed if and only if the following WHO is invertible:

$$
W_{t, \Sigma}=r_{\Sigma} A_{t} \quad: \quad H_{\Sigma}^{1 / 2} \rightarrow H^{-1 / 2}(\Sigma)
$$

In this case, the solution of the Neumann problem is given by the formulas

$$
\begin{aligned}
u & =\mathcal{K}_{N, \Omega}\left(g_{1}, g_{2}\right)=\left\{\begin{array}{lll}
\mathcal{K}_{N, \Omega^{+}} u_{1}^{+} \text {in } & \Omega^{+} \\
\mathcal{K}_{N, \Omega^{-}} u_{1}^{-} & \text {in } & \Omega^{-}
\end{array}\right. \\
\mathcal{K}_{N, \Omega^{+}} u_{1}^{+}(x) & =\mathcal{F}_{\xi^{\prime} \mapsto x^{\prime}}^{-1} e^{-t\left(\xi^{\prime}\right) x_{3}} \frac{-1}{t\left(\xi^{\prime}\right)} \widehat{u_{1}^{+}}\left(\xi^{\prime}\right) \\
& =\frac{1}{(2 \pi)^{2}} \int_{\mathbb{R}^{2}} e^{-i \xi^{\prime} x^{\prime}-t\left(\xi_{1}, \xi_{2}\right) x_{3}} \frac{-1}{t\left(\xi_{1}, \xi_{2}\right)} \widehat{u_{1}^{+}}\left(\xi_{1}, \xi_{2}\right) d \xi_{1} d \xi_{2} \\
\mathcal{K}_{N, \Omega^{-}} u_{1}^{-}(x) & =\mathcal{F}_{\xi^{\prime} \mapsto x^{\prime}}^{-1} e^{t\left(\xi^{\prime}\right) x_{3}} \frac{1}{t\left(\xi^{\prime}\right)} \widehat{u_{1}^{-}}\left(\xi^{\prime}\right) \\
& =\frac{1}{(2 \pi)^{2}} \int_{\mathbb{R}^{2}} e^{-i \xi^{\prime} x^{\prime}+t\left(\xi_{1}, \xi_{2}\right) x_{3}} \frac{1}{t\left(\xi_{1}, \xi_{2}\right)} \widehat{u_{1}^{-}}\left(\xi_{1}, \xi_{2}\right) d \xi_{1} d \xi_{2}
\end{aligned}
$$




$$
\left(\begin{array}{l}
u_{1}^{+} \\
u_{1}^{-}
\end{array}\right)=\Upsilon_{N}^{-1}\left(\begin{array}{cc}
A_{t} W_{t, \Sigma}^{-1} & 0 \\
0 & \ell_{0}
\end{array}\right) \Upsilon_{N}\left(\begin{array}{l}
g^{+} \\
g^{-}
\end{array}\right), \quad \Upsilon_{N}=\left(\begin{array}{cc}
I & I \\
I & -I
\end{array}\right) .
$$

Proof. Conclusions are similar as before.

Remark 2.5. Existence and uniqueness of a solution are known from [18, 43] in case of Lipschitz domains and from [25, 38] in case of half-planes. Hence uniqueness is trivial for convex PCDs and obvious for PCDs from (1.9).

The uniqueness result follows also directly from the Green formula, while existence can be shown with the Lax-Milgram Lemma (see, e.g., [9], Section $6.3)$.

On the other hand, by using the representation formulas of solutions with layer potentials and Plemelji-Sokhotskii formulas, one reduces both, the Dirichlet and the the Neumann BVPs to boundary integral equations with positive definite symbol in the Bessel potential spaces where the operators act in the spaces $\widetilde{H}^{1 / 2}(\Sigma) \rightarrow H^{-1 / 2}(\Sigma)$ or $\widetilde{H}^{-1 / 2}(\Sigma) \rightarrow H^{1 / 2}(\Sigma)$ (cf. (2.6) and (2.8)). Those can also be studied with the help of results from [12] (lifting in the Bessel potential spaces and pseudo-differential operators with locally sectorial symbols).

We shall see later (in the proof of Theorem 5.1) that the crucial terms in these formulas $A_{t^{-1}} W_{t^{-1}, \Sigma}^{-1}$ and $A_{t} W_{t, \Sigma}^{-1}$ can be interpreted as very particular extension operators or operators of the form of a composition $\Pi \ell$ where $\Pi$ is a particular projector and $\ell$ an arbitrary extension of a functional from $H^{ \pm 1 / 2}(\Sigma)$ to a functional in $H^{ \pm 1 / 2}$.

\section{Some results on general Wiener-Hopf operators}

A general Wiener-Hopf operator (also abbreviated by WHO) is given by

$$
W=\left.P_{2} A\right|_{P_{1} X}
$$

where $A: X \rightarrow Y$ is a bounded linear operator acting in Banach spaces and $P_{1} \in \mathcal{L}(X), P_{2} \in \mathcal{L}(Y)$ are projectors, i.e., $P_{j}^{2}=P_{j}, j=1,2$. By convention, $W$ is regarded as an operator from $P_{1} X=\operatorname{im} P_{1}$ into $P_{2} Y=\operatorname{im} P_{2}$, although, $P_{2}$ acts into $Y$, i.e., strictly speaking, not into $P_{2} Y$ (cf. [27, Chapter III]). This convention will be applied and referred to in the sequel for convenience (and following the tradition). For practical reasons we enlarge the convention by identifying in some formulas $W$ with $P_{2} A P_{1}$ as an operator acting between the full spaces. Occasionally we will also consider $W^{-1}$ as acting on the full space, i.e., we consider $P_{1} W^{-1} P_{2}=P_{1}\left(\left.P_{2} A\right|_{P_{1} X}\right)^{-1} P_{2}$. This makes some formulas more compact.

The notation (3.1) was introduced in [10,33], first in a symmetric setting where $X=Y$ and $P_{1}=P_{2}=P$ for operators in Hilbert spaces, and later in the asymmetric setting of $(3.1)[36,37]$. Main objective in those publications was the (generalized) inversion of $W$ by an operator factorization of $A$ (assuming that $A$ is invertible). Here we study a completely different (abstract) idea, presented by A.F. dos Santos [30, 31], originally connected to more special applications [24, 25, 39]. 


\subsection{Identification of general Wiener-Hopf operators}

The connection between classical WHOs (2.1) and general WHOs (3.1) is given via a continuous extension operator

$$
E_{\Sigma}^{s} \quad: \quad H^{s}(\Sigma) \rightarrow H^{s}\left(\mathbb{R}^{n}\right)
$$

provided it exists for $\Sigma \subset \mathbb{R}^{n}$ (see Section 1), namely by the identification

$$
\begin{aligned}
X=H^{r} & , \quad Y=H^{s} \\
P_{1} X=H_{\Sigma}^{r} & , \quad P_{2}=E_{\Sigma}^{s} r_{\Sigma} \\
A=\mathcal{F}^{-1} \phi \cdot \mathcal{F} & : \quad H^{r} \rightarrow H^{s} .
\end{aligned}
$$

We observe, in the identification of a general WHO, that not the full definitions of $P_{1}$ and $P_{2}$ are relevant but only im $P_{1}$ and $\operatorname{ker} P_{2}$. The domain $\operatorname{dom} W$ of $W$ is a complemented subspace of $X$ and can be seen as the image of any projector $P$ with the same image $\operatorname{im} P=\operatorname{dom} W=\operatorname{im} P_{1}$ (and arbitrary complement for the kernel), i.e.,

$$
P P_{1}=P_{1}, P_{1} P=P .
$$

Further, if $P_{2}$ and $\Pi$ are projectors with the same kernel, then the following two WHOs are equivalent:

$$
W=\left.P_{2} A\right|_{P_{1} X} \sim \widetilde{W}=\left.\Pi A\right|_{P_{1} X}=\left.\Pi A\right|_{P X}
$$

because

$$
\Pi P_{2}=\Pi, P_{2} \Pi=P_{2} .
$$

In the classical case, $\Pi$ reflects the variety of possible extension operators. In Hilbert spaces we conclude easily the following interesting result:

Proposition 3.1. Let $W=\left.P_{2} A\right|_{P_{1} X}$ be a general WHO (see (3.1)) where $X, Y$ are Hilbert spaces. Then

$$
W \sim \widetilde{W}=\left.\Pi A\right|_{P X}
$$

where $P$ and $\Pi$ are orthogonal projectors.

Proof. It is well known that the orthogonal projectors $P$ onto $P_{1} X$ and $\Pi$ along $\left(I-P_{2}\right) Y$ exist. Hence we have the equivalence relation

$$
W=\left.P_{2} A\right|_{P_{1} X}=\left.\left.P_{2} \Pi A\right|_{P X} P\right|_{P_{1} X}=\left.\left.P_{2}\right|_{\Pi Y} \widetilde{W} P\right|_{P_{1} X}
$$

between $W$ and $\widetilde{W}$ where the outer factors are bijective in the sense of the above-mentioned convention.

The foregoing result will be used in Section 4 for the construction of certain non-orthogonal projectors (in the case $k \notin i \mathbb{R}$ ) based upon the knowledge of corresponding orthogonal projectors with the same image (resulting from Lemma 4.2), by an approximation argument.

Focusing on (generalized) inverses, we obtain in a similar way: 
Proposition 3.2. Let $W, \widetilde{W}$ be general WHOs related by (3.4)-(3.6) and let $\widetilde{W}^{-}: \Pi Y \rightarrow P X$ be a generalized inverse of $\widetilde{W}$. Then a generalized inverse of $W$ is given by

$$
W^{-}=\left.\left.P_{1}\right|_{P X} \widetilde{W}^{-} \Pi\right|_{P_{2} Y} .
$$

Proof. By verification $W W^{-} W=W$.

Clearly the statement includes the cases of one-sided invertibility, Fredholmness, and invertibility that is needed in this paper.

Now, the identification of the general WHO $W=\left.P_{2} A_{\phi}\right|_{P_{1} X}$ with the Eskin type $\mathrm{WHO}$ is given by the equivalence relations

$$
E_{\Sigma}^{s} W_{\phi, \Sigma}=W \quad, \quad W_{\phi, \Sigma}=r_{\Sigma} W .
$$

Consequently, in case of invertible WHOs

$$
W_{\phi, \Sigma}^{-1}=W^{-1} E_{\Sigma}^{s} \quad, \quad A_{\phi} W_{\phi, \Sigma}^{-1}=A_{\phi} W^{-1} E_{\Sigma}^{s}
$$

which makes the connection with Theorems 2.3 and 2.4.

Another relationship between WHOs turns out to be very important in what follows. Given a general WHO (3.1), where now $A$ is assumed to be boundedly invertible, and let the complemented projectors be denoted by $Q_{1}=I_{X}-P_{1}$ and $Q_{2}=I_{Y}-P_{2}$, respectively, we call

$$
W_{*}=\left.Q_{1} A^{-1}\right|_{Q_{2} Y} \quad: \quad Q_{2} Y \rightarrow Q_{1} X
$$

the WHO associated with $W$. This notation was introduced in [10] for symmetric and in [36] for asymmetric setting, respectively. In a different context (realization theory, minimal factorization) it was called an "indicator" of $W$, thinking of various possibilities of extending $W$ to an operator matrix

$$
A=\left(\begin{array}{cc}
W & * \\
* & *
\end{array}\right),
$$

see $[1,2]$ for details.

\subsection{A geometric perspective}

Following an idea of A.F. dos Santos [30, 31] (which has roots in [10, 34] and [24]) we study a "geometric relation" between $A P_{1} X$ and $Q_{2} Y$. In contrast to the existing literature we shall base this consideration upon the following result, which seems to be still unpublished [2] but very efficient.

Lemma 3.3. Given two pairs of complementary projectors in Banach spaces, $P_{1}, Q_{1}=I-P_{1} \in \mathcal{L}(X), P_{2}, Q_{2}=I-P_{2} \in \mathcal{L}(Y)$ and an invertible operator $A \in \mathcal{L}(X, Y)$, the following operator factorization is valid:

$$
\begin{aligned}
\left(\begin{array}{cc}
P_{2} A P_{1} & 0 \\
0 & Q_{2}
\end{array}\right)= & \left(\begin{array}{cc}
P_{2} A P_{1} & 0 \\
Q_{2} A P_{1} & Q_{2}
\end{array}\right)\left(\begin{array}{cc}
P_{1} & 0 \\
-Q_{2} A P_{1} & Q_{2}
\end{array}\right) \\
= & \left(\begin{array}{cc}
P_{2} A P_{1} & P_{2} A Q_{1} \\
Q_{2} A P_{1} & Q_{2} A Q_{1}
\end{array}\right)\left(\begin{array}{cc}
P_{1} & 0 \\
0 & Q_{1} A^{-1} Q_{2}
\end{array}\right) \\
& \left(\begin{array}{cc}
P_{1} & P_{1} A^{-1} Q_{2} \\
0 & Q_{2}
\end{array}\right)\left(\begin{array}{cc}
P_{1} & 0 \\
-Q_{2} A P_{1} & Q_{2}
\end{array}\right)
\end{aligned}
$$


Proof. The formula can be verified easily.

Remark 3.4. Note that the matrix operator on the left of (3.11) acts as

$$
M: P_{1} X \oplus Q_{2} Y \rightarrow P_{2} Y \oplus Q_{2} Y \cong Y,
$$

i.e., not from $X$ into $Y$ in contrast to the first factor of the second line. For the first line of (3.11) we do not need the invertibility of $A$. It would be more adequate to write $\left.P_{2} A\right|_{P_{1} X}$ instead of $P_{2} A P_{1}$ etc. We avoided this just for cosmetic reasons (see the convention).

Theorem 3.5 (Ferreira dos Santos 1988). Let $W$ be a general WHO, given by (3.1) where $A$ is injective. Then $W$ is invertible if and only if $A P_{1} X$ and $Q_{2} Y$ are complemented subspaces of $Y$, in brief

$$
A P_{1} X \oplus Q_{2} Y=Y \text {. }
$$

If $A$ is invertible we equivalently have

$$
P_{1} X \oplus A^{-1} Q_{2} Y=X .
$$

Proof. Formula (3.12) is an interpretation of the first part of (3.11) if one takes into account that $P_{2} A P_{1}+Q_{2} A P_{1}=A P_{1}$ and that the last factor in the first line of (3.11) is invertible. The second conclusion (3.13) is then evident.

Corollary 3.6. Let $W=\left.P_{2} A\right|_{P_{1} X}: P_{1} X \rightarrow P_{2} Y$ be a general WHO with $A: X \rightarrow Y$ being invertible. Assume that $W$ is invertible (or, equivalently (3.12) holds). Then the inverse $W^{-1}: P_{2} Y \rightarrow P_{1} X$ can be represented by

$$
\begin{aligned}
W^{-1} & =\left.A^{-1} \Pi\right|_{P_{2} Y} \quad \text { where } \Pi \text { projects onto } A P_{1} X \text { along } Q_{2} Y \\
& =\left.P A^{-1}\right|_{P_{2} Y} \quad \text { where } P \text { projects onto } P_{1} X \text { along } A^{-1} Q_{2} Y .
\end{aligned}
$$

Moreover, in this case these projectors are given by

$$
\Pi=A W^{-1} P_{2} \quad, \quad P=W^{-1} P_{2} A
$$

as operators acting in $Y$ and $X$, respectively.

Remark 3.7. For the symmetric setting $\left(X=Y, P_{1}=P_{2}=P\right)$ Theorem 3.5 was proved in [10]. There are further generalizations to the case where $W$ is Fredholm, e.g., see [30,31]. Another generalization to the case where $W$ is generalized invertible might be possible by ideas of [37], we suppose. However, they are not needed here.

The formulas (3.14) imply that $P=A^{-1} \Pi A$, i.e., it obviously suffices to construct one of the two projectors $P$ and $\Pi$ in order to invert $W$.

\subsection{Matrical coupling}

Let us recall two definitions and a few known results. Two bounded linear operators in Banach spaces $S \in \mathcal{L}\left(X_{1}, Y_{1}\right), T \in \mathcal{L}\left(X_{2}, Y_{2}\right)$ are said to be matrically coupled, if there is an invertible operator matrix (with suitable entries $*$ ) such that

$$
\left(\begin{array}{ll}
S & * \\
* & *
\end{array}\right)=\left(\begin{array}{ll}
* & * \\
* & T
\end{array}\right)^{-1} .
$$


Two bounded linear operators in Banach spaces $S$ and $T$ are said to be equivalent after extension, in brief

$$
S \stackrel{*}{\sim} T
$$

if there exist Banach spaces $Z_{1}, Z_{2}$ and linear homeomorphisms $E, F$ such that

$$
\left(\begin{array}{cc}
S & 0 \\
0 & I_{Z_{1}}
\end{array}\right)=E\left(\begin{array}{cc}
T & 0 \\
0 & I_{Z_{2}}
\end{array}\right) F .
$$

Example. Looking at Lemma 3.3 and Remark 3.4 we can interpret the first line of (3.11) as

$$
W \stackrel{*}{\sim}\left(A P_{1} \mid Q_{2}\right) \quad: \quad P_{1} X \oplus Q_{2} Y \rightarrow Y
$$

In fact, the relation presented in the first line of (3.11) may be viewed as a particular form of an equivalence after extension relation since the extension is made from one side only. Namely, we have there

$$
W \oplus I_{Q_{2}} \sim\left(A P_{1} \mid Q_{2}\right),
$$

which is a so-called equivalence after one-sided extension. The equivalence after one-sided extension concept, being stronger than the equivalence after extension, is intimately related with the Schur coupling notion [5, 32]. Schur coupled operators allow even more direct relationships between their null spaces and range spaces than in the equivalence after extension relation (cf. [3, §2-3] for extra details and still existing open problems within the Schur coupling theory).

We also would like to point out that in [46], Chapter 0 by S. Puntanen and G.P.H. Styan, we may find a very pleasant historical introduction about the Schur complement. There, the last formula of page 4 presents in fact an equivalence after one-sided extension which yields a very direct proof of the famous Schur (determinant) lemma [32].

Theorem 3.8 (Bart-Tsekanovsky 1991). Let $S$ and $T$ be bounded linear operators in Banach spaces. Then $S \stackrel{*}{\sim} T$ if and only if $S$ and $T$ are matrically coupled.

Remark 3.9. The importance of this theorem for us consists in the consequence, that an inverse of $T$ can be computed from an inverse of $S$ (and vice versa, if $E^{-1}$ and $F^{-1}$ are known). This is obvious from (3.18) but not from (3.16) - and was a celebrated fact in the 1980s [1].

The sufficiency ("if") part was already proved in [1], the necessity part ("only if") later in [4].

However it was observed in [2] that the sufficiency part in the symmetric case (where $X=Y, P_{1}=P_{2}$ ) is an interpretation of a well known formula, see, e.g., $[17,29]$

$$
\begin{aligned}
P A P+Q & =(A P+Q)(I-Q A P)=A\left(P+A^{-1} Q\right)(I-Q A P) \\
& =A\left(P+Q A^{-1} Q\right)\left(I+P A^{-1} Q\right)(I-Q A P) .
\end{aligned}
$$


In the asymmetric case, it is a consequence of the formula (3.11), which can be regarded as a direct generalization of (3.21).

At the end, the second (necessity) part of Theorem 3.8 is not so evident, particularly the construction of a coupling relation from an equivalence after extension relation, see [4]. However the conclusion that is most important in our applications can be proved independently and more directly as follows.

Theorem 3.10 (Speck 1985). Let $S$ and $T$ be bounded linear operators in Banach spaces which are matrically coupled, i.e., $S=W=\left.P_{2} A\right|_{P_{1} X}$ and $T=W_{*}=\left.Q_{1} A^{-1}\right|_{Q_{2} Y}$ in the above notation. Further let $V$ be a generalized inverse of $W$, i.e., $W V W=W$. Then a generalized inverse of $W_{*}$ is given by

$$
V_{*}=\left.Q_{2}\left(A-A P_{1} V P_{2} A\right)\right|_{Q_{1} X} .
$$

Proof. Formula (3.22) is a consequence of the first part of Theorem 3.8 (matrically coupled operators are equivalent after extension) and formula (3.11) that implies: a generalized inverse of $W$ yields a generalized inverse of the matrix on the left which yields a generalized inverse of the second factor of the second line which yields a generalized inverse of $W_{*}$.

An earlier detailed and independent proof can be found in [37], p. 21-22. Note that the present proof is constructive. In [37] the formula (3.22) was just guessed and verified. Clearly it includes the cases of one-sided invertibility, Fredholmness, and invertibility that is needed here.

\section{Construction of the projectors $P$ and $\Pi$ onto/along $H_{\Sigma}^{ \pm 1 / 2}$}

In order to determine the WHO inverses needed in Theorem 2.3 and 2.4, we are now going to calculate the corresponding projectors (related by Corollary 3.6). Clearly all these operators exist and are unique as seen before in the introduction and in Theorem 3.5.

For convenience let us recall the relevant notation. Actually there appear various sceneries: (1) The abstract setting (with orthogonal and nonorthogonal projectors), (2) the concrete realizations (of Section 3.1) where $\Sigma$ is an E-domain (with two cases concerning the Dirichlet and the Neumann problem, respectively), and (3) the special situations where $\Sigma$ has particular form (half-plane, convex PCD, etc.). In the abstract setting we continue to consider the projectors

$$
\begin{array}{ccccc}
\Pi & \text { onto } & A P_{1} X & \text { along } & Q_{2} Y \\
P & \text { onto } & P_{1} X & \text { along } & A^{-1} Q_{2} Y
\end{array}
$$

where $A \in \mathcal{L}(X, Y)$ is boundedly invertible, $P_{1} \in \mathcal{L}(X), P_{2} \in \mathcal{L}(Y)$ arbitrary projectors and $Q_{1}=I_{X}-P_{1}, Q_{2}=I_{Y}-P_{2}$. Using partly the same letters (for identification) we further consider the following realization where $\Sigma$ is 
an E-domain, $s \in \mathbb{R}$ and

$$
\begin{aligned}
& A=A_{t^{2 s}}=\mathcal{F}^{-1} t^{2 s} \cdot \mathcal{F}: H^{s}\left(\mathbb{R}^{2}\right) \rightarrow H^{-s}\left(\mathbb{R}^{2}\right) \\
& P_{1} \text { is any projector in } H^{s}\left(\mathbb{R}^{2}\right) \text { onto } H_{\Sigma}^{s} \\
& P_{2} \text { is any projector in } H^{-s}\left(\mathbb{R}^{2}\right) \text { along } H_{\Sigma^{\prime}}^{-s} .
\end{aligned}
$$

For the Dirichlet problem we have $A=A_{t^{-1}}$, i.e. $s=-1 / 2$, for the Neumann problem $A=A_{t}$, i.e., $s=1 / 2$. However, many of the following considerations work for general $s \in \mathbb{R}$.

The simplest case appears when $\Sigma$ is a half-plane and $k=i$. Here we obtain formulas in closed analytical form and orthogonal projectors as follows:

Example. Consider the half-plane $\Sigma=\mathbb{R}_{1+}^{2}=\left\{x \in \mathbb{R}^{2}: x_{1}>0\right\}$, the (orthogonal) projectors $P_{+}=\ell_{0} r_{+}: L^{2}\left(\mathbb{R}^{2}\right) \rightarrow L^{2}\left(\mathbb{R}^{2}\right)$ onto $L_{\Sigma}^{2}=L_{\mathbb{R}_{1+}^{2}}^{2}$, $P_{-}=I-P_{+}$and the Bessel potential operators $[11,12,16]$ of order $s \in \mathbb{R}$ :

$$
\begin{array}{ll}
\Lambda_{+}^{s}=A_{\lambda_{+}^{s}}, & \lambda_{+}^{s}(\xi)=\left(\xi_{1}+i \sqrt{\xi_{2}^{2}+1}\right)^{s}, \xi \in \mathbb{R}^{2} \\
\Lambda_{-}^{s}=A_{\lambda_{-}^{s}}, & \lambda_{-}^{s}(\xi)=\left(\xi_{1}-i \sqrt{\xi_{2}^{2}+1}\right)^{s}, \xi \in \mathbb{R}^{2} .
\end{array}
$$

For any $s \in \mathbb{R}$ we find the orthogonal projectors [7]

$$
\begin{aligned}
P_{+}^{s} & =\Lambda_{+}^{-s} P_{+} \Lambda_{+}^{s} \text { onto } H_{\Sigma}^{s} \\
P_{-}^{s} & =\Lambda_{-}^{-s} P_{-} \Lambda_{-}^{s} \text { onto } H_{\Sigma^{\prime}}^{s} \\
\Pi_{+}^{s} & =\Lambda_{-}^{-s} P_{+} \Lambda_{-}^{s} \text { along } H_{\Sigma^{\prime}}^{s} \\
\Pi_{-}^{s} & =\Lambda_{+}^{-s} P_{-} \Lambda_{+}^{s} \text { along } H_{\Sigma}^{s} .
\end{aligned}
$$

Hence $P_{+}^{s}+\Pi_{-}^{s}=I_{H^{s}}$ and $P_{-}^{s}+\Pi_{+}^{s}=I_{H^{s}}$.

If we specify $\Sigma=\mathbb{R}_{1+}^{2}=\left\{x \in \mathbb{R}^{2}: x_{1}>0\right\}$ and $k=i$ in the second scenery, then $P_{1}=P_{+}^{s}$ and $P_{2}=\Pi_{+}^{-s}$ satisfy (4.2). If we specify moreover $A=A_{t^{2 s}}$ in the first scenery, then $P=P_{1}=P_{+}^{s}$ and $\Pi=P_{2}=\Pi_{+}^{-s}$ satisfy (4.1).

The projector $\ell_{0} r_{+}$in $L^{2}\left(\mathbb{R}^{2}\right)$ coincides with the multiplication by the characteristic functions $\chi_{+}$of the half space $\mathbb{R}_{1,+}^{2}$. This observation can be generalized to $\ell_{0} r_{\Sigma}$ acting in the spaces $H^{s}\left(\mathbb{R}^{2}\right)$ if $|s|<1 / 2$ provided $\Sigma$ is a Lipschitz domain or an E-domain in $\mathbb{R}^{2}$, e.g., instead of the half space $\mathbb{R}_{1,+}^{2}$.

\subsection{Preliminaries}

First we mention two facts which are independent of the choice of $\Sigma$. The first is a consequence of (1.12), the second is known from [19].

Lemma 4.1. The projectors $\Pi, P$ of (4.1) in the situation (4.2) are orthogonal with respect to the inner product (1.12) if $k=i$.

Proof. Orthogonality of the two projectors is equivalent to the fact that

$$
\left\langle A_{t} \varphi, \psi\right\rangle_{-s}=0 \text { for } \varphi \in H_{\Sigma}^{s}, \psi \in H_{\Sigma^{\prime}}^{-s} .
$$


It suffices to consider smooth, rapidly decreasing functions, which are dense in these spaces, $\varphi \in \mathcal{S}_{\Sigma}=\{\varphi \in \mathcal{S}: \operatorname{supp} \varphi \subset \bar{\Sigma}\}$ and $\psi \in \mathcal{S}_{\Sigma^{\prime}}$. These must satisfy

$$
\int_{\mathbb{R}^{2}} A_{\phi} \varphi(\xi) \overline{\psi(\xi)} d \xi=0 \text { where } \phi=\left(\frac{t(\xi)}{\lambda(\xi)}\right)^{2 s}=\frac{\left(\xi^{2}-k^{2}\right)^{s}}{\left(\xi^{2}+1\right)^{s}} .
$$

Now supp $A_{\phi} \varphi \subset \bar{\Sigma}$ for all $\varphi \in \mathcal{S}_{\Sigma}$ with $\operatorname{supp} \varphi \subset \bar{\Sigma}$ obviously holds if $k=i$, i.e., $t=\lambda$.

Lemma 4.2 (Halmos 1982, Problem 96). Given any Hilbert space $H$ and orthogonal projectors $p_{1}, p_{2}, \ldots, p_{m} \in \mathcal{L}(H)$, the orthogonal projector onto $\operatorname{im} p_{1} \cap \operatorname{im} p_{2}$ is given by the so-called infimum of the two projectors:

$$
p_{1} \wedge p_{2}=\prod_{j=1}^{\infty}\left(p_{1} p_{2}\right)^{j}=\lim _{N \rightarrow \infty} \prod_{j=1}^{N}\left(p_{1} p_{2}\right)^{j}
$$

which converges strongly. The orthogonal projector $p$ onto $\operatorname{im} p_{1} \cap \ldots \cap \operatorname{im} p_{m}$ is given by

$$
p=p_{1} \wedge \ldots \wedge p_{m}=\wedge_{j=1}^{m} p_{j}
$$

that is defined by iteration and represents an associative operation.

\subsection{Case $k=i$, convex PCDs}

In this section we assume $A_{t}=\Lambda=A_{\lambda}$, i.e., $k=i$ (see (1.13)) and use the following brief notation. For any open half-plane $\Sigma \subset \mathbb{R}^{2}$ let

$$
M_{\Sigma} \quad: \quad \Sigma \rightarrow \mathbb{R}_{1+}^{2}=\left\{x \in \mathbb{R}^{2}: x_{1}>0\right\}
$$

be the canonical linear transformation that transforms $\Sigma$ onto $\mathbb{R}_{1+}^{2}$, i.e., by a minimal dilation plus a rotation in the mathematical positive sense, say. Moreover let

$$
J_{\Sigma} f(x)=f\left(M_{\Sigma} x\right) \quad, \quad x \in \Sigma \text { or } x \in \mathbb{R}^{2},
$$

for functions and distributions defined on $\Sigma$ or defined on $\mathbb{R}^{2}$, as well.

Theorem 4.3. Let $\Sigma$ be a convex PCD, i.e., $\Sigma=\cap_{j=1}^{m} \Sigma_{j}$ with half-planes $\Sigma_{j} \subset \mathbb{R}^{2}, j=1, \ldots, m$ and $s \in \mathbb{R}$. Then the orthogonal projector $P_{\Sigma}^{s}$ onto $P_{1} X=H_{\Sigma}^{s}$ projects along $\Lambda^{-2 s} H_{\Sigma^{\prime}}^{-s}$ and is given by

$$
\begin{aligned}
P_{\Sigma}^{s} & =\wedge_{j=1}^{m} P_{\Sigma_{j}}^{s} \\
P_{\Sigma_{j}}^{s} & =J_{\Sigma_{j}}^{-1} P_{+}^{s} J_{\Sigma_{j}} \quad, \quad j=1, \ldots, m .
\end{aligned}
$$

The orthogonal projector $\Pi$ onto $\Lambda^{2 s} H_{\Sigma}^{s}$ projects along $Q_{2} Y=H_{\Sigma^{\prime}}^{-s}$ and is given by

$$
\Pi_{\Sigma}^{-s}=\Lambda^{2 s} P_{\Sigma}^{s} \Lambda^{-2 s}
$$

Proof. As $H_{\Sigma}^{s}$ is a closed subspace of the Hilbert space $H^{s}$, it is complemented and the orthogonal projector onto $H_{\Sigma}^{s}$ exists and is unique. Lemma 4.2 implies that the orthogonal projector onto $H_{\Sigma}^{s}$ is given by formula (4.7). 
Every projector $P_{\Sigma_{j}}^{s}$ projects along $\Lambda^{-2 s} H_{\Sigma_{j}^{\prime}}^{-s}$. Since $\Sigma=\bigcap \Sigma_{j}$, the orthogonal projector onto $H_{\Sigma}^{s}$ projects along $\Lambda^{-2 s} H_{\Sigma^{\prime}}^{-s}$, because $\Sigma^{\prime}=\operatorname{int} \bigcup \overline{\Sigma_{j}^{\prime}}$.

The second part of the theorem with formula (4.8) is a consequence of the first part, exchanging the roles of $\Sigma$ and $\Sigma^{\prime}$, of $s$ and $-s$, and thinking of the complementary projector (exchanging "onto" and "along").

\subsection{Case $k=i$, arbitrary PCDs}

Theorem 4.4. Let $\Sigma \subset \mathcal{A}_{2}$, i.e., $\Sigma=\operatorname{int} \bigcup_{j=1, \ldots, n}$ clos $\Sigma_{j}$ where $\Sigma_{j}$ are convex PCDs, and assume that $s \in \mathbb{R}$. Then the orthogonal projector $P_{\Sigma}^{s}$ onto $H_{\Sigma}^{s}$ projects along $\Lambda^{-2 s} H_{\Sigma^{\prime}}^{-s}$, i.e., $P_{\Sigma}^{s}=I-\Pi_{\Sigma^{\prime}}^{s}$ and is given by

$$
P_{\Sigma}^{s}=I-\wedge_{j=1}^{m} \Pi_{\Sigma_{j}^{\prime}}^{s}=I-\wedge_{j=1}^{m}\left(I-P_{\Sigma_{j}}^{s}\right)
$$

with $P_{\Sigma_{j}}^{s}$ taken from Theorem 4.3 (representing each $\Sigma_{j}$ as intersection of half-planes).

Proof. The assumption $\Sigma \subset \mathcal{A}_{2}$ implies that $\Sigma^{\prime}=\bigcap_{j=1}^{m} \Sigma_{j}^{\prime}$ where $\Sigma_{j}^{\prime}=$ $\mathbb{R}^{2} \backslash \overline{\Sigma_{j}}$. Looking at $(4.9), P_{\Sigma_{j}}^{s}$ projects onto $H_{\Sigma_{j}}^{s}$ along $\Lambda^{-2 s} H_{\Sigma_{j}^{\prime}}^{-s}$. Hence $I-P_{\Sigma_{j}}^{s}$ projects along $H_{\Sigma_{j}}^{s}$ onto $\Lambda^{-2 s} H_{\Sigma_{j}^{\prime}}^{-s}$ and $\wedge\left(I-P_{\Sigma_{j}}^{s}\right)$ is the orthogonal projector onto $\bigcap\left(\Lambda^{-2 s} H_{\Sigma_{j}^{\prime}}^{-s}\right)=\Lambda^{-2 s} \bigcap H_{\Sigma_{j}^{\prime}}^{-s}$, thus projecting along $H_{\Sigma}^{s}$. This implies that $P_{\Sigma}^{s}$ as given by (4.9) projects orthogonally onto $H_{\Sigma}^{s}$.

4.4. Case $k \in i \mathbb{R}_{+}$, i.e., $\Re e k=0$, ऽmk $>0$

In this section we show that the previous results remain valid for $k \in i \mathbb{R}_{+}$if we change the topology to another equivalent one. I.e., we remain in the same Hilbert spaces but infinite series and infinite products converge in a different sense, with respect to a modified norm.

Definition 4.5. Let $H^{s, k}\left(\mathbb{R}^{n}\right)$ be the space that coincides with $H^{s}\left(\mathbb{R}^{n}\right)$ as a linear space equipped with the form

$$
\begin{aligned}
\langle\varphi, \psi\rangle_{s, k} & =\left\langle A_{t}^{s} \varphi, A_{t}^{s} \psi\right\rangle_{0} \\
& =\int_{\mathbb{R}^{n}} A_{t}^{s} \varphi(x) \cdot \overline{A_{t}^{s} \psi(x)} d x \\
& =\int_{\mathbb{R}^{n}} \widehat{\varphi}(\xi) \widehat{\psi}(\xi)\left|\xi^{2}-k^{2}\right|^{s} d \xi .
\end{aligned}
$$

Proposition 4.6. For any $k \in \mathbb{C} \backslash \mathbb{R}, H^{s, k}\left(\mathbb{R}^{n}\right)$ is a Hilbert space with norm

$$
\|\varphi\|_{s, k}=\langle\varphi, \varphi\rangle_{s, k}^{1 / 2}
$$

that is equivalent to the norm of $H^{s}\left(\mathbb{R}^{n}\right)$.

Proof. It is a consequence of the fact that $A_{t}^{s}: H^{s}\left(\mathbb{R}^{n}\right) \rightarrow L^{2}\left(\mathbb{R}^{n}\right)$ is a linear homeomorphism.

Remark 4.7. Obviously we have $H^{s, i}\left(\mathbb{R}^{n}\right)=H^{s}\left(\mathbb{R}^{n}\right)$. The spaces $H^{s, k}(\Sigma)$, $\widetilde{H}^{s, k}(\Sigma), H_{\Sigma}^{s, k}$ may be defined by analogy to the spaces where $k=i$. Evidently the analogue of Proposition 4.6 holds for these spaces, as well. 
Remark 4.8. The spaces $H^{s}\left(\mathbb{R}^{n}\right)=H^{s, i}\left(\mathbb{R}^{n}\right)$ and $H^{s, k}\left(\mathbb{R}^{n}\right)$ are isomorphic for all $k \in i \mathbb{R}$ and the norms in these spaces are equivalent, because both of them are isomorphic to $L^{2}\left(\mathbb{R}^{n}\right)$. The isomorphism is performed by the Fourier convolution operator $A_{\omega_{s, k}}=\mathcal{F}^{-1} \omega_{s, k} \mathcal{F}$ with the symbol

$$
\omega_{s, k}(\xi):=\left(\frac{\xi^{2}-k^{2}}{\xi^{2}+1}\right)^{s}
$$

which is invertible by $A_{\omega_{s, k}^{-1}}$.

Proposition 4.9. For any domain $\Sigma$, any number $n=2,3, \ldots$ and $s \in \mathbb{R}$ the subspaces $A_{t}^{2 s} H_{\Sigma}^{s, k}$ and $H_{\Sigma^{\prime}}^{-s, k}$ where $\Sigma^{\prime}=\operatorname{int}\left(\mathbb{R}^{2} \backslash \Sigma\right)$ are orthogonal to each other if $k \in i \mathbb{R}_{+}$.

Proof. The algebraic decomposition

$$
A_{t}^{2 s} H_{\Sigma}^{s, k} \dot{+} H_{\Sigma^{\prime}}^{-s, k}=H^{-s, k}
$$

is clear from the case $k=i$, see Section 3.2. By definition $H_{\Sigma}^{s, k}$ and $H_{\Sigma^{\prime}}^{-s, k}$ are closed subspaces and, for $\varphi \in H_{\Sigma}^{s, k}, \psi \in H_{\Sigma^{\prime}}^{-s, k}$, we have

$$
\begin{aligned}
\left\langle A_{t}^{2 s} \varphi, \psi\right\rangle_{-s, k} & =\left\langle A_{t}^{s} \varphi, A_{t}^{-s} \psi\right\rangle_{0} \\
& =\int_{\mathbb{R}^{n}} \widehat{\varphi}(\xi)\left(\xi^{2}-k^{2}\right)^{s / 2} \overline{\widehat{\psi}(\xi)\left(\xi^{2}-k^{2}\right)^{-s / 2}} d \xi
\end{aligned}
$$

which disappears for any such pair $\varphi, \psi$ if $k^{2}$ is real.

Evidently, the analogue of Theorem 4.4 for $k \in i \mathbb{R}_{+}$instead of $k=i$ is valid, as well.

Corollary 4.10. Let $\Sigma$ be a PCD, $k \in i \mathbb{R}_{+}$and $s \in \mathbb{R}$. Then the following orthogonal projectors can be presented explicitly:

$$
\begin{aligned}
P_{\Sigma}^{s} & =I-\wedge_{j=1}^{m} \Pi_{\Sigma_{j}^{\prime}}^{s}=I-\wedge_{j=1}^{m}\left(I-P_{\Sigma_{j}}^{s}\right) \text { onto } H_{\Sigma}^{s} \\
P_{\Sigma^{\prime}}^{s} & =I-A_{t}^{-2 s} P_{\Sigma}^{-s} A_{t}^{2 s} \text { onto } H_{\Sigma^{\prime}}^{s} \\
\Pi_{\Sigma}^{s} & =A_{t}^{-2 s} P_{\Sigma}^{-s} A_{t}^{2 s} \quad \text { along } H_{\Sigma^{\prime}}^{s} \\
\Pi_{\Sigma^{\prime}}^{s} & =I-P_{\Sigma}^{s} \text { along } H_{\Sigma}^{s}
\end{aligned}
$$

where the decomposition of $\Sigma$ from Theorem 4.3 is used and the infinite products converge in the sense of the norm (4.11).

4.5. Case $\Re e k \neq 0, \Im m k>0$

Now a modification of the scalar product (4.10) does not help anymore. Thus we shall use a different idea coming up from [24] to present the (nonorthogonal) projectors $\Pi_{\Sigma}^{ \pm 1 / 2}$ etc. by Neumann series approximation using the orthogonal projectors constructed before. Therefore we extend the notation by

$\Pi_{\Sigma, k}^{s} \quad-$ the projector onto $A_{t^{-2 s}} H_{\Sigma}^{-s}$ along $H_{\Sigma^{\prime}}^{s}$

$\Pi_{\Sigma, i}^{s} \quad-$ the projector onto $\Lambda^{-2 s} H_{\Sigma}^{-s}$ along $H_{\Sigma^{\prime}}^{s}$ 
where the latter coincides with the first for $k=i$ and is orthogonal. Further projectors can be defined and treated by analogy. So we have:

$$
\begin{aligned}
& P_{\Sigma, k}^{s}-\text { the projector onto } H_{\Sigma}^{s} \text { along } A_{t^{-2 s}} H_{\Sigma^{\prime}}^{-s} \\
& P_{\Sigma, k}^{s}=I-\Pi_{\Sigma^{\prime}, k}^{s}
\end{aligned}
$$

which we employ basically for $s= \pm 1 / 2$ and call them briefly $\Pi$-projectors and P-projectors.

Proposition 4.11. Abbreviate $\Pi=\Pi_{\Sigma, i}^{1 / 2}$. Then the projector $\Pi_{\Sigma, k}^{1 / 2}$ is given by

$$
\Pi_{\Sigma, k}^{1 / 2}=A_{t^{-1}} \Lambda W_{0}^{-1} \Pi \quad, \quad W_{0}=\left.\Pi A_{t^{-1}} \Lambda\right|_{\Pi H^{1 / 2}}
$$

(using the convention) where the inverse $W_{0}^{-1}$ is given by a Neumann series. Proof. The operator $W_{0}=\left.\Pi A_{t^{-1}} \Lambda\right|_{\Pi H^{1 / 2}}$ is invertible by a Neumann series, since $\Re e A_{t^{-1}} \Lambda>0$ and $\Pi$ is orthogonal, see [10, 24, 37].

The operator $\Pi_{\Sigma, k}^{1 / 2}$ in (4.16) is obviously linear, bounded and idempotent, hence it is a projector. $W_{0}^{-1}$ maps onto $\Pi H^{1 / 2}=\Lambda^{-1} H_{\Sigma}^{-1 / 2}$, thus $A_{t^{-1}} \Lambda W_{0}^{-1} \Pi$ maps onto $A_{t^{-1}} H_{\Sigma}^{-1 / 2}$. Finally its kernel is obviously $H_{\Sigma^{\prime}}^{-s}$ and the proof is complete.

The following result is analogous.

Proposition 4.12. Abbreviate $\Pi=\Pi_{\Sigma, i}^{-1 / 2}$. Then the projector $\Pi_{\Sigma, k}^{-1 / 2}$ is given by

$$
\Pi_{\Sigma, k}^{-1 / 2}=A_{t} \Lambda^{-1} W_{0}^{-1} \Pi \quad, \quad W_{0}=\left.\Pi A_{t} \Lambda^{-1}\right|_{\Pi H^{-1 / 2}}
$$

(using the convention) where the inverse $W_{0}^{-1}$ is given by a Neumann series.

\subsection{Equivalent constructions}

Here we show that the construction of various involved operators is equivalent, i.e., they can be obtained easily from each other. As a matter of fact, this has nothing to do with the form of $\Sigma$ nor with Wiener-Hopf factorization, but with the features exposed in Sections 3.2 and 3.3: the geometric perspective and matrical coupling.

Theorem 4.13. Let $\Sigma$ be an E-domain. Consider the Dirichlet problem for $\Sigma$ (as described in (1.3)-(1.5)) and the Neumann problem for $\Sigma^{\prime}$ (by analogy). Then the two resolvent operators (see (2.7), (2.9)), the WHOs therein, and corresponding P-projectors and П-projectors (see (4.14), (4.15)) can be computed from each other.

Proof. Clearly each of the P-projectors yield the corresponding $\Pi$-projector by definition, see (4.15), and the corresponding WHO inverses by Corollary 3.6 which yield the corresponding resolvent operators, see Theorem 2.3 and Theorem 2.4. For clarity we summarize these very direct relations:

$$
\begin{gathered}
P_{\Sigma, k}^{-1 / 2}=I-\Pi_{\Sigma^{\prime}, k}^{-1 / 2}=W_{t^{-1}, \Sigma}^{-1} r_{\Sigma} A_{t^{-1}} \quad \text { onto } H_{\Sigma}^{-1 / 2} \quad \text { along } A_{t} H_{\Sigma^{\prime}}^{1 / 2} \\
P_{\Sigma^{\prime}, k}^{1 / 2}=I-\Pi_{\Sigma, k}^{1 / 2}=W_{t, \Sigma^{\prime}}^{-1} r_{\Sigma} A_{t} \quad \text { onto } H_{\Sigma^{\prime}}^{1 / 2} \quad \text { along } A_{t^{-1}} H_{\Sigma}^{-1 / 2}
\end{gathered}
$$


according to the WHO inverses ( - appearing in)

$$
\begin{gathered}
W_{t^{-1}, \Sigma}^{-1}=P_{\Sigma, k}^{-1 / 2} A_{t} \ell-\text { Dirichlet problem for } \Sigma \\
W_{t, \Sigma^{\prime}}^{-1}=P_{\Sigma^{\prime}, k}^{1 / 2} A_{t^{-1}} \ell-\text { Neumann problem for } \Sigma^{\prime} .
\end{gathered}
$$

Herein $\ell$ denotes any extension operator from $H^{1 / 2}(\Sigma)$ into $H^{1 / 2}\left(\mathbb{R}^{2}\right)$ or from $H^{-1 / 2}(\Sigma)$ into $H^{-1 / 2}\left(\mathbb{R}^{2}\right)$, respectively.

Conversely, from the resolvent operators we obtain the corresponding projectors and WHO inverses by composition with trace and symmetrization operators, e.g., $W_{t^{-1}, \Sigma}^{-1}: g \mapsto u \mapsto u_{0}^{+}-u_{0}^{-}$etc. (see Theorem 2.3 and Theorem $2.4)$.

Now the geometric perspective (see Theorem 3.5 and Corollary 3.6) implies that the projectors in the above list are related by

$$
P_{\Sigma, k}^{-1 / 2}=A_{t} \Pi_{\Sigma, k}^{1 / 2} A_{t^{-1}} .
$$

An alternative proof, instead of using (4.18), can be based upon the fact that the two WHOs in the above scheme, $W_{t^{-1}, \Sigma}^{-1}$ and $W_{t, \Sigma^{\prime}}^{-1}$, are matrically coupled (see Lemma 3.3). Again the relationship with the resolvent operators is evident from the representation formulas.

Exchanging the roles of $\Sigma$ and $\Sigma^{\prime}$ we observe that the operators corresponding to the Dirichlet problem for $\Sigma^{\prime}$ and the Neumann problem for $\Sigma$ are related in a similar way.

\section{Explicit solution of the BVPs}

We come to the final results in concrete form presenting the details of the proof of Theorem 1.3.

Theorem 5.1. Let $\Sigma \subset \mathbb{R}^{2}$ be a PCD and $k \in \mathbb{C}, \Im m k>0$. The resolvent operators for the Dirichlet and Neumann problems are given by Theorem 2.3 and Theorem 2.4, respectively, where

$$
\begin{gathered}
W_{t^{-1}, \Sigma}^{-1}=A_{t} \Pi_{\Sigma, k}^{1 / 2} \ell=P_{\Sigma, k}^{-1 / 2} A_{t} \ell: H^{1 / 2}(\Sigma) \rightarrow H_{\Sigma}^{-1 / 2} \\
W_{t, \Sigma}^{-1}=A_{t}^{-1} \Pi_{\Sigma, k}^{-1 / 2} \ell=P_{\Sigma, k}^{1 / 2} A_{t}^{-1} \ell: \quad H^{-1 / 2}(\Sigma) \rightarrow H_{\Sigma}^{1 / 2}
\end{gathered}
$$

with arbitrary extension operators $\ell$ into $H^{1 / 2}$ or $H^{-1 / 2}$, respectively, and the projectors are also explicitly given in

- Theorem 4.3 for convex PCDs and $k=i$,

- Theorem 4.4 for arbitrary PCDs and $k=i$,

- Corollary 4.9 for arbitrary PCDs and $k \in i \mathbb{R}_{+}$,

- Proposition 4.10 for arbitrary PCDs, ऽmk $>0$, the Dirichlet problem,

- Proposition 4.11 for arbitrary PCDs, ऽmk >0, the Neumann problem.

Proof. All that results directly from the previous as referred to. 
Remark 5.2. There are few cases where the resolvent operators can be obtained in closed analytical form (in a representation without infinite series and products). Half-plane screens represent one of them, see Section 4. The possibility of applying factorization methods to other screen problems with conical configurations is not positively answered till now in the authors opinion (in contrast to wedge problems, see $[8,14,15,41]$ ).

Let us consider the special geometrical case where $\Sigma$ is a cone, moreover connected and convex. V.B. Vasil'ev proposed in his book [44] to solve the diffraction problem by use of a so-called wave factorization of the function $t(\xi)=\left(\xi^{2}-k^{2}\right)^{1 / 2}$ into two factors, holomorphic in certain tube domains. However, looking at the explicit form of the two factors, it turns out that they vanish within the corresponding tube domains, see [44], pages 28-29 and 3839. This means that the given factorization is not a wave factorization in the sense of the author's own Definition 5.1 and therefore not helpful for the solution of the problem. The authors of the present article do not know any other example from mathematical physics where the method of wave factorization is applicable.

Other canonical screen problems such as the diffraction from a flat circular disc (see [21, 45] for instance) end up with Fredholm integral equations and series expansion, as well, but not with a solution in closed analytic form.

In view of the complexity of the derived formulas, some simplification can be obtained for a screen that is complementary to a convex PCD by the following corollaries. The idea is known as a sort of abstract Babinet principle [39].

Corollary 5.3. Let $\Sigma$ be an E-domain. Assume that the inverse of $W_{t}, \Sigma$ is known (which provides the resolvent to the Neumann problem for $\Sigma$ by Theorem 2.4). Then the Dirichlet problem for $\Sigma^{\prime}$ is uniquely solved by Theorem 2.4 where $W_{t^{-1}, \Sigma^{\prime}}^{-1}$ is obtained from Theorem 3.10, substituting $V=W_{t, \Sigma}^{-1}$ and the corresponding other terms.

Corollary 5.4. Let $\Sigma$ be an E-domain. Assume that the inverse of $W_{t^{-1}, \Sigma}$ is known (which provides the resolvent to the Dirichlet problem for $\Sigma$ by Theorem 2.3). Then the Neumann problem for $\Sigma^{\prime}$ is uniquely solved by Theorem 2.3 where $W_{t, \Sigma^{\prime}}^{-1}$ is obtained from Theorem 3.10, substituting $V=W_{t^{-1}, \Sigma}^{-1}$ and the corresponding other terms.

We finish with a result on the low regularity of solutions to the BVPs.

Theorem 5.5. Let $\Sigma \subset \mathbb{R}^{2}$ be a $P C D$, ऽmk $>0$ and $\left.\varepsilon \in\right] 0,1 / 2[$.

I. If $g \in H^{1 / 2+\varepsilon}(\Sigma)_{\sim}^{2}$ and $u \in \mathcal{H}^{1}(\Omega)$ is a solution of the Dirichlet problem for $\Sigma$, then $u \in \mathcal{H}^{1+\varepsilon}(\Omega)$, i.e., $u^{ \pm} \in H^{1+\varepsilon}\left(\Omega^{ \pm}\right)$, see (1.2)-(1.5). Moreover the resolvent operator $(2.7)$ restricted to these spaces represents a linear homeomorphism:

$$
\mathcal{K}_{D, \Omega}^{\varepsilon}: H^{1 / 2+\varepsilon}(\Sigma)_{\sim}^{2} \rightarrow \mathcal{H}^{1+\varepsilon}(\Omega) .
$$

II. If $g \in H^{-1 / 2+\varepsilon}(\Sigma)^{2}$ and $u \in \mathcal{H}^{1}(\Omega)$ is a solution of the Neumann problem for $\Sigma$, then $u \in \mathcal{H}^{1+\varepsilon}(\Omega)$, i.e., $u^{ \pm} \in H^{1+\varepsilon}\left(\Omega^{ \pm}\right)$, see (1.2)-(1.5). Moreover the 
resolvent operator (2.7) restricted to these spaces represents a linear homeomorphism:

$$
\mathcal{K}_{N, \Omega}^{\varepsilon} \quad: \quad H^{-1 / 2+\varepsilon}(\Sigma)^{2} \rightarrow \mathcal{H}^{1+\varepsilon}(\Omega) .
$$

Proof. Following all the way long the foregoing construction of resolvent operators we realize that there is no problem to include the parameter $\varepsilon \in] 0,1 / 2[$. Notice that there is no compatibility condition in the second statement II since $\widetilde{H}^{-1 / 2+\varepsilon}(\Sigma)=H^{-1 / 2+\varepsilon}(\Sigma)$ for $\left.\varepsilon \in\right] 0,1 / 2[$.

\section{Open problems}

At the end we would like to formulate some unsolved problems that we found interesting.

Problem 6.1. How can we treat other boundary conditions like impedance, oblique derivative etc.? It is known that these BVPs lead to WHOs of the form $W_{\phi, \Sigma}=r_{\Sigma} A_{\phi}$ where the Fourier symbol $\phi$ is more complicated and a matrical coupling relation and orthogonal projectors can not be seen.

Problem 6.2. In non-Hilbert spaces $W^{s, p}$ we have no orthogonality. Is there any alternative approach, perhaps working with sesquilinear forms (as a generalization of the Halmos Theorem)?

Problem 6.3. Replacing the Helmholtz equation by the Lamé or Maxwell equations, can we obtain analogous results considering matrix WHOs?

Problem 6.4. Slit domains and cracks may be tackled by certain space modifications. Are there any interesting new results, techniques, applications?

Problem 6.5. Arbitrary convex screens could be formally treated by an infinite product $\wedge_{j=1}^{\infty} P_{j}$ considering $\Sigma$ as an intersection of infinitely many half-planes. What about convergence?

\section{Acknowledgment}

The work was supported in part by FCT - Portuguese Foundation for Science and Technology through the Center for Functional Analysis and Applications at Instituto Superior Técnico, Universidade Técnica de Lisboa, and through the Center for Research and Development in Mathematics and Applications (PEst-C/MAT/UI4106/2011) at University of Aveiro. The authors would like to thank the organizers of the conference WOAT 2012, Prof. Amélia Bastos et al., for their invitations leading to the possibility to continue our fruitful cooperation.

\section{References}

[1] H. Bart, I. Gohberg and M. Kaashoek, The coupling method for solving integral equations. Oper. Theory Adv. Appl. 2 (1984), 39-73.

[2] H. Bart, I. Gohberg and M. Kaashoek, Addendum to: The coupling method for solving integral equations. Integr. Equ. Oper. Theory 8 (1985), 890-891. 
[3] H. Bart, I. Gohberg, M.A. Kaashoek, A.C.M. Ran, Schur complements and state space realizations. Linear Algebra Appl. 399 (2005), 203-224.

[4] H. Bart and V. E. Tsekanovskii, Matricial coupling and equivalence after extension. Oper. Theory Adv. Appl. 59 (1991), 143-160.

[5] H. Bart, V.E. Tsekanovskii, Complementary Schur complements. Linear Algebra Appl. 197 (1994), 651-658.

[6] L.P. Castro and F.-O. Speck, Regularity properties and generalized inverses of delta-related operators. Z. Anal. Anwend. 17 (1998), 577-598.

[7] L.P. Castro and F.-O. Speck, Relations between convolution type operators on intervals and on the half-line. Integr. Equ. Oper. Theory 37 (2000) 169-207.

[8] L.P. Castro, F.-O. Speck and F.S. Teixeira, On a class of wedge diffraction problems posted by Erhard Meister. Oper. Theory Adv. Appl. 147 (2004), 211238.

[9] P.G. Ciarlet, Mathematical Elasticity III: Theory of Shells. Studies in Mathematics and Applications 29. Elsevier, North-Holland, Amsterdam, 2000.

[10] A. Devinatz and M. Shinbrot, General Wiener-Hopf operators. Trans. AMS 145 (1969), 467-494.

[11] R. Duduchava and F.-O. Speck, Bessel potential operators for the quarter-plane. Appl. Anal. 45 (1992), 49-68.

[12] R. Duduchava and F.-O. Speck, Pseudodifferential operators on compact manifolds with Lipschitz boundary. Math. Nachr. 160 (1993), 149-191.

[13] R. Duduchava, W. Wendland, The Wiener-Hopf method for systems of pseudodifferential equations with an application to crack problems. Integr. Equ. Oper. Theory 23 (1995), 294-335.

[14] T. Ehrhardt, A.P. Nolasco and F.-O. Speck, Boundary integral methods for wedge diffraction problems: the angle $2 \pi / n$, Dirichlet and Neumann conditions. Operators and Matrices 5 (2011), 1-40.

[15] T. Ehrhardt, A.P. Nolasco and F.-O. Speck, A Riemann surface approach for diffraction from rational angles. Operators and Matrices, to appear, $55 \mathrm{p}$.

[16] G.I. Eskin, Boundary Value Problems for Elliptic Pseudodifferential Equations. American Mathematical Society, Providence, Rhode Island, 1981.

[17] I.Z. Gochberg and I.A. Feldman, Faltungsgleichungen und Projektionsverfahren zu ihrer Lösung (in German). Birkhäuser and Akademie-Verlag, Berlin, 1974 (Russian edition in 1971).

[18] P. Grisvard, Elliptic Problems in Non-Smooth Domains. Pitman, London, 1985.

[19] P.R. Halmos, A Hilbert Space Problem Book. Second edition, Springer, New York, 1982.

[20] G.C. Hsiao and W.L. Wendland, Boundary Integral Equations. Springer, Berlin, 2008.

[21] D.S. Jones, Diffraction at frequencies by a circular disc. Proc. Camb. Philos. Soc. 61 (1965), 223-245.

[22] M.G. Krein, Integral equations on a half-line with kernel depending upon the difference of the arguments. AMS Transl., Ser. 2, 22 (1962), 163-288.

[23] V.G. Maz'ya and S.V. Poborchi, Differentible Functions in Bad Domains. World Scientific, Singapure, 1997. 
[24] E. Meister and F.-O. Speck, Scalar diffraction problems for Lipschitz and polygonal screens. Z. Angew. Math. Mech. 67 (1987), 434-435.

[25] E. Meister and F.-O. Speck, A contribution to the quarter-plane problem in diffraction theory. J. Math. Anal. Appl. 130 (1988), 223-236.

[26] E. Meister and F.-O. Speck, Modern Wiener-Hopf methods in diffraction theory. Ordinary and Partial Differential Equations 2, Pitman Res. Notes Math. Ser. 216 (Longman, London, 1989), 130-171.

[27] S.G. Mikhlin and S. Prössdorf, Singular Integral Operators. Extended and partly modified translation from the German by A. Böttcher and R. Lehmann. Springer, Berlin, 1986 (German edition by Akademie-Verlag, Berlin, 1980).

[28] A. Moura Santos, F.-O. Speck and F.S. Teixeira, Minimal normalization of Wiener-Hopf operators in spaces of Bessel potentials. J. Math. Anal. Appl. 225 (1998), 501-531.

[29] S. Prössdorf, Some Classes of Singular Equations. North-Holland, Amsterdam, 1978 (German edition by Akademie-Verlag, Berlin, 1974).

[30] A.F. dos Santos, Abstract Wiener-Hopf Operators - Geometric Perspective. Thesis on the occasion of "Provas de Agregação" (in Portuguese), Departamento de Matemática, Instituto Superior Técnico, Universidade Técnica de Lisboa, Lisbon, 1988.

[31] A.F. dos Santos, General Wiener-Hopf operators and representation of their generalized inverses. Oper. Theory Adv. Appl. 41 (1989), 473-483.

[32] I. Schur, Über Potenzreihen, die im Innern des Einheitskreises beschränkt sind. I, II. J. für Math. 147 (1917), 205-232; 148 (1918), 122-145 (in German).

[33] M. Shinbrot, On singular integral operators. J. Math. Mech. 13 (1964), 395-406.

[34] M. Shinbrot, On the range of general Wiener-Hopf operators. J. Math. Mech. 18 (1969), 587-601.

[35] S.G. Sommerfeld, Mathematische Theorie der Diffraction. Math. Annalen 47 (1896), 317-374.

[36] F.-O. Speck, On the generalized invertibility of Wiener-Hopf operators in Banach spaces. Integr. Equ. Oper. Theory 6 (1983), 458-465.

[37] F.-O. Speck, General Wiener-Hopf Factorization Methods. Pitman, London, 1985.

[38] F.-O. Speck, Mixed boundary value problems of the type of Sommerfeld's halfplane problem. Proc. R. Soc. Edinb., Sect. A 104 (1986), 261-277.

[39] F.-O. Speck, Diffraction from a three-quarter-plane using an abstract Babinet principle. Z. Ang. Math. Mech. 93 (2013), 485-491.

[40] F.-O. Speck, On the reduction of linear systems related to boundary value problems. Oper. Theory Adv. Appl. 228 (2013), 391-406.

[41] F.-O. Speck and E. Stephan, Boundary value problems for the Helmholtz equation in an octant. Integr. Equ. Oper. Theory 62 (2008), 269-300.

[42] E. Stein, Singular Integrals and Differentiability Properties of Functions. Princeton University Press, Princeton, N.J., 1970.

[43] E. Stephan, Boundary Integral Equations for Mixed Boundary Value Problems, Screen and Transmission Problems in $\mathbb{R}^{3}$. Habilitation thesis, Preprint 848, Fachbereich Mathematik, Technische Hochschule Darmstadt, 1984. 
[44] V.B. Vasil'ev, Wave Factorization of Elliptic Symbols: Theory and Applications. Kluver, Dordrecht, 2000.

[45] S.S. Vinogradov, A method for the solution of a problem of diffraction on a thin disc. Dokl. Akad. Nauk Ukr. SSR, Ser. A 1983, No.6 (1983), 37-40 (in Russian).

[46] F. Zhang (ed.), The Schur Complement and its Applications. Numerical Methods and Algorithms 4. Springer-Verlag, New York, 2005.

Luís P. Castro

Departamento de Matemática

Universidade de Aveiro

Aveiro

Portugal

e-mail: castro@ua.pt

Roland Duduchava

Andrea Razmadze Mathematical Institute

Ivane Javakhishvili Tbilisi State University

Tbilisi

Georgia

e-mail: dudu@rmi.ge

Frank-Olme Speck

Instituto Superior Técnico

Universidade Técnica de Lisboa

Avenida Rovisco Pais

Portugal

e-mail: fspeck@math.ist.utl.pt 